Цитирование: Нечушкина В.М., Морхов К.Ю., Егорова А.В. Выбор объема хирургического лечения у больных раком тела матки. Злокачественные опухоли 2020; 3s1: 3-10

\title{
ВЫБОР ОБЪЕМА ХИРУРГИЧЕСКОГО ЛЕЧЕНИЯ У БОЛЬНЫХ РАКОМ ТЕЛА МАТКИ
}

\author{
В.М. Нечушкина ${ }^{1,2}$, К.Ю. Морхов ${ }^{1}$, А.В. Егорова ${ }^{2}$ \\ 1. ФГБУ «НМИЦ АГП им. В.И. Кулакова» Минздрава России, Москва, Россия \\ 2. ФГАОУ ВО «РНИМУ им. Н.И. Пирогова» Минздрава России, Москва, Россия \\ Для корреспонденции: drnechushkina@mail.ru
}

Резюме: Статья посвящена вопросам хирургического лечения рака тела матки. Непрекращающиеся в течение нескольких десятилетий дискуссии относительно объема хирургических вмешательств при раке тела матки ранних стадий теперь распространились и на хирургическое лечение диссеминированных форм заболевания. В статье обсуждаются показания к лимфаденэктомии и ее объем, перспективы циторедуктивных операций при диссеминированном раке тела матки и ряд других вопросов.

Ключевые слова: рак тела матки, тазовая лимфаденэктомия, поясничная лимфаденэктомия, адъювантная лучевая терапия, циторедуктивные операции.

Рак тела матки (РТМ) составляет 4,4\% злокачественных опухолей у женщин и занимает 6-е место в структуре заболеваемости женщин злокачественными опухолями в мире [25]. Пожилой возраст больных, высокая частота ожирения и других сопутствующих заболеваний объясняют непрекращающиеся дискуссии, касающиеся тактики лечения больных РТМ. Хорошо известно, что хирургический метод является основой лечения РТМ и не имеет эффективных альтернатив. По данным Международной федерации акушеров и гинекологов (FIGO), 5-летняя общая выживаемость при проведении лучевой терапии (Лт) по радикальной программе при РТМ составляет только 48,6\% [4; 21]. Однако у 5-15\% больных операция невозможна из-за тяжелых сопутствующих заболеваний [36]. Хирургическое лечение РТМ претерпело значительные изменения. Расширяются объемы хирургических вмешательств не только при ранних, но и при поздних стадиях болезни.

\section{ХИРУРГИЧЕСКОЕ ЛЕЧЕНИЕ РТМ РАННИХ СТАДИЙ}

Основные дискуссии, которые не прекращаются на протяжении по крайней мере последних 30 лет, касаются оценки состояния регионарных лимфатических узлов при РТМ I клинической стадии, на долю которого приходится 70-75\% всех наблюдений РТМ [21].

В 1952 г. С.Т. Jarvet отметил, что частота поражения лимфатических узлов при РТМ достигает $28 \%$ [31]. Прогностическая значимость поражения лимфатических узлов при РТМ была впервые отмечена исследователями под руководством J.A. Stallworthy [35; 44]. Эти данные стали отправной точной для классических исследований лимфогенного метастазирования РТМ, которые были начаты в 70-е гг. прошлого столетия. В 1976 г. проф. Я.В. Бохман опубликовал данные о 400 больных РТМ, которым была выполнена расширенная экстирпация матки с придатками [1]. Он показал, что частота метастазов в тазовых лимфатических узлах растет по мере снижения степени дифференцировки опухоли, достигая 26,9\% при низкодифференцированном РТМ, а также по мере увеличения глубины инвазии миометрия, составляя $34,4 \%$ при опухолях с глубиной инвазии миометрия более 1 см. Кроме того, было отмечено неблагоприятное влияние на прогноз опухолей с переходом на канал шейки матки: частота поражения тазовых лимфатических узлов при них достигла 27,6\% [1].

За рубежом классическим считается исследование Онкогинекологической исследовательской группы GOG 33 [20]. В это исследование была включена 621 больная РТМ I клинической стадии. Распространение опухоли за пределы матки (метастазы в лимфатических узлах или яичниках, диссеминация по брюшине или опухолевые клетки в смывах из брюшной полости) выявлено у $22 \%$ больных РТМ I клинической стадии. Метастазы в тазовыхи/или поясничных лимфатическихузлах обнаружены у $11 \%$ больных, поражение тазовых и поясничных лимфатических узлов-у $3 \%$, изолированное поражение только поясничных лимфатических узлов-у $2 \%$. Таким образом, частота метастазов в тазовых лимфатических узлах при РТМ I клинической стадии составила $9 \%$, в поясничных$5 \%$, частота поражения поясничных лимфатических узлов 
при наличии метастазов в тазовых - $38 \%$. Вслед за проф. Я.В. Бохманом авторы исследования GOG 33 отметили повышение риска лимфогенного метастазирования РТМ при снижении дифференцировки, увеличении глубины инвазии, переходе опухоли на шейку матки. Кроме того, было продемонстрировано неблагоприятное прогностическое влияние поражения придатков матки и других вариантов выхода опухоли за пределы матки, а также наличия опухолевых клеток в смывах из брюшной полости и опухолевых эмболов в лимфатических щелях [20].

В этом же исследовании авторы впервые подчеркнули два важнейших принципа выполнения лимфаденэктомии при РТМ: (1) она должна быть адекватной для суждения о состоянии лимфатических узлов; (2) хирург не должен определять показания к лимфаденэктомии по результатам интраоперационной пальпации лимфатических узлов, поскольку макрометастазы выявляются менее чем у $10 \%$ больных РТМ с лимфогенными метастазами, а пальпация регионарных лимфатических узлов через брюшину зачастую не позволяет выявить их изменения даже при наличии метастазов [20].

Вопросы адекватности лимфаденэктомии при РТМ подробно изучались позднее и другими авторами. Было показано, что чем больше число удаленных лимфатических узлов, тем точнее оценка распространения опухоли. В исследование J.K. Chan и соавт. были включены 11443 больные PTM I - III стадий, данные о которых были включены в базу данных SEER (база данных Национального института рака США) [15]. Увеличение числа удаленных лимфатических узлов было сопряжено с большей вероятностью выявления их метастатического поражения. Согласно результатам этого исследования, при единичных метастазах удаление 21-25 лимфатических узлов статистически значимо повышало вероятность их обнаружения. Удаление более 25 лимфатических узлов не приводило к дальнейшему росту частоты выявления метастатически измененных лимфатических узлов [15].

В настоящее время мы располагаем результатами двух рандомизированных исследований, посвященных изучению прогностической роли лимфаденэктомии при РТМ ранних стадий, причем оба исследования не выявили повышения выживаемости больных РТМ, которым была выполнена тазовая лимфаденэктомия. Первым следует упомянуть исследование MRC ASTEC, проведенное группой ASTEC (A Study in the Treatment of Endometrial Cancer изучение результатов лечения РТМ) [10]. В это многоцентровое исследование были включены 1408 больных РТМ I клинической стадии. После рандомизации 704 больным выполнено стандартное вмешательство (экстирпация матки с придатками, смывы из брюшной полости, пальпация лимфатических узлов), еще у 704 больных стандартное вмешательство было дополнено тазовой лимфаденэктомией. Затем больные с промежуточным и высоким риском прогрессирования РТМ (низкодифференцированные опухоли IA - IB стадий, IC стадия по классификации FIGO
1988 г., вовлечение эндоцервикальных желез, папиллярный и светлоклеточный рак), за исключением больных с верифицированными метастазами в лимфатических узлах, были включены в рандомизированное исследование адъювантного дистанционного облучения малого таза (ДОМТ). Медиана периода наблюдения составила $37 \mathrm{mec.} \mathrm{Абсолютные}$ различия 5-летней общей выживаемости составили $1 \%$ в пользу стандартного лечения, 5-летней безрецидивной выживаемости - 6\% в пользу стандартного лечения [10].

Еще одно рандомизированное исследование тазовой лимфаденэктомии при РТМ проведено в Италии [11]. В него были включены 514 больных РТМ I клинической стадии. После рандомизации 250 больным была выполнена экстирпация матки с придатками, у 264 больных она была дополнена тазовой лимфаденэктомией. Медиана числа удаленных лимфатических узлов была существенно выше, чем в исследовании MRC ASTEC, и составила 30. Тазовая лимфаденэктомия статистически значимо повышала точность определения стадии РТМ: метастазы в лимфатических узлах выявлены у 13,3\% больных в группе лимфаденэктомии и лишь у 3,2\% больных в группе без лимфаденэктомии ( $<<0,001)$. Медиана периода наблюдения составила 49 мес. Не было отмечено статистически значимых различий 5-летней безрецидивной и общей выживаемости больных, которые составили 81,0 и 85,9\% в группе лимфаденэктомии и 81,7 и 90,0\% в группе без лимфаденэктомии соответственно. Поясничную лимфаденэктомию в этом исследовании выполняли по усмотрению хирурга (она была выполнена 26\% больных, у $10 \%$ из них выявлены метастазы в поясничных лимфатических узлах). Аналогичным образом, по усмотрению лечащего врача, назначали послеоперационную Лт. Группы не различались по частоте проведения адъювантной ЛТ, однако как она влияла на выживаемость больных, не известно, поскольку она не была стандартизована [11].

Можно ли доказать влияние лимфаденэктомии на выживаемость больных РТМ? По-видимому, да. В 2012 г. были опубликованы результаты ретроспективного анализа из базы данных SEER. В него были включены 56360 больных PTM I стадии [17]. На первом этапе больным выполняли экстирпацию матки с придатками, 41,6\% больных выполнена лимфаденэктомия. К группе высокого риска относили больных низкодифференцированным РТМ ІВ стадии (FIGO, 2009 г.), к группе промежуточного риска больных умеренно-или низкодифференцированным РТМ либо РТМ IB стадии (FIGO, 2009 г.) 70 лет и старше, а также больных умереннодифференцированным РТM IB стадии (FIGO, 2009 г.) 50 лет и старше. К группе низкого риска относили всех остальных больных. В результате группу низкого риска составили 70,4\% (n=39 666), группу промежуточного риска $-26,2 \%(n=14753)$, группу высокого риска $-3,4 \%$ больных ( $\mathrm{n}=1941)$. Оказалось, что лимфаденэктомия статистически значимо повышала выживаемость больных РТМ во всех группах, в том числе больных, которым не проводили лТ. Однако абсолютные различия общей вы- 
живаемости в группе низкого риска составили лишь $1 \%$, что вряд ли можно считать клинически значимыми [17]. Именно большое число больных РТМ с низким риском прогрессирования затрудняет проведение рандомизированных исследований при этой патологии. Так, если на долю I стадии заболевания приходится около $70 \%$ больных всех больных РТМ, а на долю группы низкого риска прогрессирования - примерно 70\% больных РТМ І стадии, то низкую вероятность прогрессирования и, следовательно, благоприятный прогноз имеет половина всех больных этой патологией $[21 ; 23]$.

Еще один важный вопрос, касающийся выполнения лимфаденэктомии при РТМ,- это ее объем. Для РТМ описаны две зоны регионарного лимфооттока - тазовые и поясничные лимфатические узлы. Тактика удаления только тазовых лимфатических узлов при РТМ привлекает своей относительной технической простотой и безопасностью. Однако клиническим значением поражения поясничных лимфатических узлов при этой патологии пренебрегать нельзя. Данные исследования GOG 33, демонстрирующие, что частота изолированного поражения поясничных лимфатических узлов при РТМ I клинической стадии составляет лишь $2 \%$, часто рассматриваются вне контекста [20]. На практике лимфаденэктомию больным с крайне низким риском поражения лимфатических узлов обычно не выполняют. Таким образом, у больных, которым лим $\phi$ аденэктомия планируется, риск изолированного поражения поясничных лимфатических узлов оказывается выше. Индивидуальная величина риска зависит от совокупности прогностических факторов. Сегодня надежных данных для ее оценки нет. В целом среди больных РТМ с лимфогенными метастазами частота поражения поясничных лимфатических узлов составляет 26-66\%, а частота их изолированного поражения 5-21\% [20; 28; 29; 32; 37; 38; $40 ; 42]$. Если удалены только тазовые лимфатические узлы и в них есть метастазы, то вероятность поражения поясничных лимфатических узлов составляет 30-40\% [20; 28; $32 ; 37 ; 38 ; 42]$, а по некоторым данным достигает $60 \%$ [40].

Крайне интересными представляются результаты японского исследования SEPAL (Survival Effect of Para-Aortic Lymphadenectomy in endometrial cancer - влияние поясничной лимфаденэктомии на выживаемость больных РТМ), опубликованные в 2010 г. [47]. В это нерандомизированное исследование была включена 671 больная РТМ, получавшая лечение в двух японских онкологических центрах. В одном центре больным выполняли тазовую лимфаденэктомию, в другом - тазовую и поясничную. В группах промежуточного и высокого риска прогрессирования РТМ после операции проводили химиотерапию или лт. Общая выживаемость больных, которым выполняли тазовую и поясничную лимфаденэктомию, была статистически значимо выше таковой больных, которым выполняли только тазовую лимфаденэктомию [отношение рисков (ОР) 0,53; $95 \%$ доверительный интервал (ДИ) 0,38-0,76; $\mathrm{p}=0,0005]$. Та же закономерность отмечена в группе больных промежу- точного и высокого риска прогрессирования РТМ. Общая выживаемость больных с низким риском прогрессирования РТМ не зависела от объема лимфаденэктомии. Согласно результатам многофакторного анализа, при промежуточном и высоком риске прогрессирования тазовая и поясничная лимфаденэктомия статистически значимо снижала риск смерти от РТМ по сравнению с тазовой лимфаденэктомией (относительный риск (ОР) 0,44; $95 \%$ доверительный интервал (ДИ) 0,30-0,64; р <0,0001). При анализе результатов лечения 328 больных с промежуточным и высоким риском прогрессирования РТМ, которым после операции проводили адъювантную химиотерапию или лТ, показано, что общую выживаемость больных статистически значимо повышали выполнение тазовой и поясничной лимфаденэктомии и проведение химиотерапии [47].

Подводя итоги, следует подчеркнуть, что какой риск метастазов в лимфатических узлах при РТМ следует считать достаточно высоким, чтобы выполнять лимфаденэктомию, и какуюлимфаденэктомию выполнять, зависит от хирурга. По данным наших исследований, тазовая и поясничная лимфаденэктомия целесообразна при эндометриоидном РТМ I стадии с глубокой инвазией миометрия независимо от степени дифференцировки, а также при низкодифференцированном РТМ I стадии, поскольку частота поражения тазовых и поясничных лимфатических узлов при глубокой инвазии миометрия составляет 11 и $6 \%$ даже при высокой степени дифференцировки, а при низкодифференцированном эндометриоидном РТМ - 9 и $4 \%$ соответственно даже при начальной инвазии миометрия [7; 20]. По рекомендациям согласительной конференции ESMO, ESGO, ESTRO больные высоко-и умереннодифференцированным РТМ I клинической стадии с инвазией более половины толщины миометрия и больные низкодифференцированным РТМ I клинической стадии с инвазией менее половины толщины миометрия имеют промежуточный риск лимфогенных метастазов, поэтому лимфаденэктомию им можно выполнять для уточнения стадии, а можно и не выполнять [18]. На наш взгляд, в этих случаях целесообразно выполнение лимфаденэктомии у молодых больных, которым нежелательно проводить послеоперационную дистанционнуюлт $[3 ; 6 ; 8]$.

При умереннодифференцированных эндометриоидных опухолях с инвазией миометрия не более половины его толщины частота поражения тазовых лимфатических узлов составляет 5-9\%, поясничных - 0-4\% [9]. По рекомендациям согласительной конференции ESMO, ESGO, ESTRO в этой группе лимфаденэктомия не показана [18], однако нельзя забывать о вероятности снижения степени дифференцировки в окончательном гистологическом заключении, а также о трудностях макроскопической оценки глубины инвазии при умеренно-и низкодифференцированных опухолях [26].

При высокодифференцированном эндометриоидном PTM I стадии с инвазией миометрия менее чем на половину его толщины риск лимфогенных метастазов составляет 
менее $5 \%$ [20], поэтому лимфаденэктомия в этих случаях не показана. При низкой степени дифференцировки и глубокой инвазии миометрия частота метастазов в тазовых и поясничных лимфатических узлах достигает 34 и $23 \%$ соответственно [20]. В этой подгруппе РТМ I клинический стадии лимфаденэктомия показана абсолютно.

Тазовая и поясничная лимфаденэктомия показана также при серозном, светлоклеточном РТМ, смешанной аденокарциноме эндометрия I стадии, а также при РТМ II стадии, когда риск поражения регионарных лимфатических узлов существенно выше, чем при эндометриоидном РТМ I стадии. Лимфаденэктомия, безусловно, должна быть адекватной для полной оценки состояния регионарных лимфатических узлов. При наличии показаний к лимфаденэктомии удаляют лимфатические узлы обеих регионарных зон: тазовые и поясничные до уровня почечных сосудов (лимфодиссекция IV уровня) $[2 ; 5 ; 18]$.

Если факторы риска лимфогенных метастазов выявлены в послеоперационном гистологическом исследовании, а лимфаденэктомия не выполнена, может обсуждаться вопрос о повторном хирургическом вмешательстве, причем данная позиция закреплена в рекомендациях согласительной конференции ESMO, ESGO, ESTRO и рекомендациях RUSSCO [5; 18].

Вопросы выполнения лимфаденэктомии при РТМ тесно связаны с вопросами назначения адъювантной Лт. С одной стороны, большинство прогностически неблагоприятных факторов, которые при РТМ ранних стадий можно назвать «внутриматочными» (гистологический тип, степень дифференцировки и размер опухоли, а также ее переход на шейку матки) и служат показаниями для адъювантной ЛТ, реализуются посредством поражения регионарных лимфатических узлов [19]. С другой стороны, в последние годы изменились взгляды на безопасность ДОМТ.

В 2007 г. опубликован метаанализ, в который включены 7 рандомизированных исследований адъювантной лТ при РТМ [32]. Согласно полученным результатам, ДОМТ повышает риск смерти при низком риске прогрессирования РТМ (опухоли ІА стадии, высоко-и умереннодифференцированные опухоли IB стадии; FIGO, 1988 г.) (ОР смерти 0,71; $95 \%$ ДИ 0,52-0,96). ДОМТ не влияет на общую выживаемость больных при промежуточном риске прогрессирования (высоко-и умереннодифференцированный РТМ IC стадии, низкодифференцированный РТМ ІВ стадии) (ОР 0,97; 95\% ДИ 0,69-1,35). ДОМТ статистически значимо (на 10\%) повышает 5-летнюю общую выживаемость больных при высоком риске прогрессирования РТМ (низкодифференцированные опухоли IC стадии) (ОР 1,76; $95 \%$ ДИ 1,07-2,89). Кроме того, ДОМТ меняет характер прогрессирования РТМ, статистически значимо повышая риск отдаленных метастазов (ОР 1,58; $95 \%$ ДИ 1,07-2,35) [32].

Повышение риска смерти при длительном наблюдении за больными РТМ, которым проведено ДОМТ, демонстрируют и другие авторы. В 2012 г. повторно проанализированы результаты лечения больных, включенных в исследование
Aaldders J. и соавт. [9; 41]. Медиана периода наблюдения составила 21 год. Статистически значимых различий общей и безрецидивной выживаемости между группами сочетанной и внутриполостной лТ выявлено не было. ДОМТ статистически значимо снижало риск рецидивов и регионарных метастазов. Однако в возрасте моложе 60 лет ДОМТ статистически значимо повышало риск смерти (ОР 1,36; 95\% ДИ 1,06-1,76), в первую очередь за счет статистически значимо более частого развития метахронных опухолей (ОР 1,9; 95\% ДИ 1,23-3,03). В заключение авторы указали, что не следует рекомендовать ДОМТ больным РТМ I стадии, особенно в возрасте моложе 60 лет [41].

Согласно нашим данным, «критическим» возрастом для больным РТМ I стадии является 58 лет: у больных РТМ молодой возрастной группы, которым проводили дистанционную или сочетанную Лт, общая выживаемость была статистически значимо ниже, чем в группе наблюдения (ОР 1,98; $95 \%$ дИ 1,03-3,81) [3; 8].

По данным последнего кокрановского обзора, посвященного адъювантной ЛТ при РТМ I стадии, ДОМТ снижает риск рецидивов и регионарных метастазов РТМ, но не оказывает статистически значимого влияния на общую выживаемость и выживаемость, определяемую РТМ. При этом проведение ДОМТ сопряжено с клинически и статистически значимым повышением частоты осложнений и снижением качества жизни [34]. Авторы отмечают, что данных для того, чтобы судить о неэффективности дистанционной ЛТ при РТМ І стадии, относящемся к группе высокого риска прогрессирования - согласно исследованиям PORTEC и GOG 99, это низкодифференцированные опухоли IC стадии (FIGO, 1988 г.) [22; 33] - пока недостаточно [34].

Эти данные заставляют по-новому взглянуть на роль регионарной лимфаденэктомии при РТМ I стадии. Во-первых, большинство неблагоприятных прогностических факторов, которые являются показаниями для ДОМТ, одновременно являются факторами риска лимфогенных метастазов [19]. Во-вторых, проведение дистанционной и сочетанной ЛТ статистически значимо не влияет на прогрессирование РТМ I - II стадий в виде метастазов в тазовых лимфатических узлах, что позволяет считать применение ДОМТ с целью профилактики регионарных метастазов при РТМ малоэффективным [3; 8]. В-третьих, известно, что в отсутствие метастазов в лимфатических узлах у больных РТМ І стадии, которым выполнена лимфаденэктомия, рецидивы у стенки таза редки, а прогрессирование носит в основном местный или отдаленный характер [33; 45; 46]. И, наконец, в-четвертых, в исследованиях GOG 99 и PORTEC снижение частоты местнорегионарного прогрессирования в группе адъювантной дистанционной ЛТ происходило в основном за счет снижения риска рецидивов в культе влагалища, на долю которого приходилось $75 \%$ случаев местнорегионарного прогрессирования в группе наблюдения [11; 33].

Таким образом, с точки зрения профилактики регионарного прогрессирования лимфаденэктомию при РТМ I стадии можно рассматривать как альтернативу дистан- 
ционной ЛТ, более безопасную с точки зрения отдаленных последствий, что особенно важно у молодых больных. При необходимости для снижения риска местного прогрессирования можно применять внутриполостную лт. Эффективность этого метода в профилактике местного прогрессирования РТМ в сравнении с дистанционной лТ доказана в рандомизированном исследовании PORTEC-2 [39].

Трудности хирургического лечения больных РТМ и необходимость оценки состояния регионарных лимфатических узлов стимулируют изучение возможности биопсии сторожевых лимфоузлов при РТМ. Биопсия сторожевых лимфоузлов может служить компромиссом между невыполнением лимфаденэктомии и выполнением двухзональной лимфодиссекции. Появляется все больше данных о том, что биопсия сторожевых лимфоузлов при РТМ может быть целесообразна. По данным ретроспективного итальянского исследования, в котором приняли участие 802 больные РТМ I клинической стадии, биопсия сторожевых лимфоузлов статистически значимо увеличивала частоту выявления метастазов в лимфатических узлах $(16,7$ по сравнению с $7,3 \%)$, но не снижала безрецидивную выживаемость [13]. Однако до сих пор нет ни одного завершенного рандомизированного исследования биопсии сторожевых лимфоузлов при РТМ. В связи с этим в рекомендациях согласительной конференции ESMO, ESGO, ESTRO сказано, что биопсия сторожевых лимфоузлов при РТМ остается экспериментальным методом, однако довольно крупные ретроспективные исследования указывают на ее выполнимость и более высокую вероятность выявления микрометастазов и изолированных опухолевых клеток, хотя клиническое значение этих находок при РТМ неясно [18].

\section{ХИРУРГИЧЕСКОЕ ЛЕЧЕНИЕ РТМ ПОЗДНИХ СТАДИЙ}

На долю поздних стадий приходится менее $20 \%$ больных с впервые установленным диагнозом РТМ. В течение длительного времени при диссеминированном РТМ ограничивались выполнением паллиативной экстирпации матки с придатками, которая снижала риск кровотечения и позволяла без перерывов проводить ЛТ и ХТ. Однако уже в конце XX в. стали появляться работы, указывающие на то, что выживаемость больных РТМ поздних стадий зависит от объема остаточной опухоли после хирургического вмешательства.

B 2000 г. R.E. Bristow и соавт. опубликовали анализ результатов лечения 65 больных PTM IVB стадии [12]. Авторы отметили, что оптимальная циторедукция (размер наибольшей остаточной опухоли не более 1 см) оказалась возможной у 55,4\% больных. Чаще всего при РTM IVB стадии выполняли удаление большого сальника (68\%), перитонэктомию (62\%) и лимфаденэктомию (60\%). Авторы указали на статистически значимые различия медианы продолжительности жизни больных при выполнении оптимальной (34,3 мес) и неоптимальной (11,0 мес) циторедуктивной операции ( $p=0,0001)$. При отсутствии макроскопически определяемой остаточной опухоли, т. е. при выполнении полной циторедукции, медиана продолжительности жизни превысила 3 года и достигла 40,6 мес. В заключении авторы особо подчеркнули, что целью первичного хирургического лечения распространенного РТМ должна быть максимальная циторедукция [12].

В 2011 г. K.K. Shih и соавт. опубликовали еще одно небольшое ретроспективное исследование циторедуктивных операций при РTM IV стадии [43]. По данным многофакторного анализа, общую выживаемость больных определяли только два фактора: размер остаточной опухоли и проведение адъювантной химиотерапии. При этом медиана продолжительности жизни больных, прооперированных без остаточной опухоли, составила 42,2 мес, прооперированных с остаточной опухолью - 19,0 мес, неоперированных - только 2,2 мес [43].

В более крупном ретроспективном многоцентровом японском исследовании приняли участие 248 больных диссеминированным РТМ [24]. У $62 \%$ больных была отмечена диссеминация только в пределах брюшной полости, у $23 \%$ - только вне брюшной полости, у остальных $15 \%$ она носила смешанный характер. У $76 \%$ больных с распространением вне брюшной полости была поражена только одна зона, причем чаще всего (69\% больных) это были легкие. Результаты лечения, полученным японскими исследователями, оказались выше, чем опубликованные R.E. Bristow и соавт. Так, медиана продолжительности жизни в отсутствие остаточной опухоли в брюшной полости составила $48 \mathrm{мес,} \mathrm{при} \mathrm{наличии} \mathrm{остаточной} \mathrm{опухоли} \mathrm{не} \mathrm{более} 1$ см23 мес, при наличии остаточной опухоли более 1 см - 14 мес. Крайне интересными оказались результаты анализа продолжительности жизни больных в зависимости от наличия метастазов за пределами брюшной полости. При распространении только в пределах брюшной полости медиана продолжительности жизни больных после полной циторедукции составила 40 мес, при наличии остаточной опухоли - 18 мес, при наличии метастазов за пределами брюшной полости эти показатели оказались 58 и 11 мес соответственно [24].

Эти данные привели к тому, что в рекомендациях согласительной конференции ESMO, ESGO, ESTRO появился следующий пункт: при распространенном РТМ рекомендуется выполнение циторедуктивных операций без макроскопически определяемой остаточной опухоли и полноценное определение хирургической стадии [18].

Особое место занимает хирургический этап в лечении больных РТМ с метастазами в регионарных лимфатических узлах. В одной из ключевых работ, посвященных этой проблеме, опубликованной L.J. Havrilesky и соавт., по данным многофакторного анализа было показано, что неудаление макроскопически измененных лимфатических узлов при РТМ характеризуется наиболее высоким ОР смерти от основного заболевания (ОР 6,85, p =0,009) [27]. Распространение по брюшине или метастазы в яичниках 
(OP 2,24, P =0,036), пожилой возраст (OP 1,09, $\mathrm{P}<0,001)$ и поражение более двух лимфатических узлов (ОР 3,12, $\mathrm{p}=0,007$ ) оказались менее значимыми факторами риска [27].

По данным многоцентрового ретроспективного исследования, в котором участвовали больные РTM II - IV стадий, лимфаденэктомия статистически значимо повышала выживаемость, определяемую основным заболеванием, при этом у больных с лимфогенными метастазами этот показатель статистически значимо различался в зависимости от числа удаленных лимфатических узлов [30].

По данным многоцентрового ретроспективного когортного исследования TGOG-2005, 5-летняя общая выживаемость 541 больной эндометриоидным РTM III - IV стадий составила 70,4\%, медиана продолжительности жизни - 52 мес, медиана времени до прогрессирования 43 мес [16]. Как общая выживаемость, так и выживаемость без прогрессирования статистически значимо различались в группах больных, которым была выполнена селективная лимфаденэктомия, тазовая или поясничная и двухзональная лимфодиссекция [16].

В анализ базы данных SEER за период с 1988 по 2001 г. была включена 1221 больная PTM IIIC - IV стадий с метастазами в регионарных лимфатических узлах [14]. Авторы отметили статистически значимое повышение выживаемости, определяемой основным заболеванием, в зависимости от числа удаленных лимфатических узлов. Причем различия отмечались при любом числе пораженных лимфатических узлов. Так, при наличии более пяти метастазов и удалении 6-10 лимфатических узлов 5-летняя выживаемость, определяемая основным заболеванием, составила 20,9\%, при удалении 11-20 лимфатических узлов - 33,8\%, а при удалении более 20 лимфатических узлов достигала 60,5\% [14].

\section{ЗАКЛЮЧЕНИЕ}

В заключение следует отметить, что с точки зрения профилактики регионарного прогрессирования лимфаденэктомию при РТМ I стадии можно рассматривать как альтернативу дистанционной ЛТ, более безопасную с точки зрения отдаленных последствий, что особенно важно у молодых больных. Следует понимать, что лимфаденэктомия позволяет уточнить стадию болезни и облегчает планирование ЛТ. Если лимфаденэктомия выполняется, то она должна быть двухзональной, т. е. удалению подлежат тазовые и поясничные лимфатические узлы до уровня почечных сосудов. Лимфаденэктомия не показана при низком риске лимфогенных метастазов: у больных высокодифференцированным эндометриоидным РТМ I клинической стадии с инвазией не более половины толщины миометрия и абсолютно показана при низкодифференцированном РТМ I клинической стадии с инвазией более половины толщины миометрия. Остальные больные находятся в «серой зоне». Лимфаденэктомия у них может выполняться для уточнения стадии. Если факторы риска лимфогенных метастазов выявлены в послеоперационном гистологи- ческом исследовании, а лимфаденэктомия не выполнена, может обсуждаться вопрос о повторном хирургическом вмешательстве, причем данная позиция закреплена в рекомендациях согласительной конференции ESMO, ESGO, ESTRO и рекомендациях RUSSCO $[5 ; 18]$. Крайне перспективным представляется изучение онкологических результатов биопсии сторожевых лимфоузлов при РТМ, однако очевидно, что сейчас необходимы рандомизированные исследования по этой проблеме.

При РТМ поздних стадий выживаемость больных зависит от объема остаточной опухоли, причем наиболее высокая выживаемость отмечена после полной циторедукции. Лимфаденэктомия повышает выживаемость больных РТМ с метастазами в лимфатических узлах. Наиболее высокая выживаемость отмечена после тазовой и поясничной лимфодиссекции и удаления более 20 лимфатических узлов.

\section{ЛИТЕРАТУРА}

1. Бохман Я.В. Метастазы рака матки.-Л.: Медицина, 1976.159 c.

2. Клинические рекомендации Министерства здравоохранения Российской Федерации «Рак тела матки и саркомы матки 2020 [Электронныйресурс].-Москва:Министерство здравоохранения Российской Федерации, 2020.-URL:http://cr.rosminzdrav.ru/\#!/ schema/320 (дата обращения 01.10.2020).

3. Нечушкина, В.М. Рак тела матки (факторы прогноза и тактика лечения): дис. ... д-ра мед. наук: 14.01.12/Нечушкина Валентина Михайловна.-М., 2013.- 445 с.

4. Нечушкина В.М., Кузнецов В.В., Баринов В. В., Бокина Л.И. Рак тела матки/М.И. Давыдов, В.В. Кузнецов, В.М. Нечушкина (ред.) Лекции по онкогинекологии.-М.: МЕДпресс-информ, 2009.- С. 226-254.

5. Нечушкина В.М., Коломиец Л.А., Кравец О.А. и соавт. Практические рекомендации по лекарственному лечению лечению рака тела матки и сарком матки // Злокачественные опухоли.-2019.-Т. 9, Т 3 (прил. 2).-С. 218-232.

6. Нечушкина В.М., Морхов К.Ю., Кузнецов В.В. Адъювантная терапия при раке тела матки ранних стадий // Вестн. РОНЦ им. Н. Н. Блохина РАМН.-2012.- T. 23, № 4.- С. 33-40.

7. Нечушкина В.М., Морхов К.Ю., Кузнецов В.В. Тазовая и поясничная лимфаденэктомия при раке тела матки // Вестн. РОНЦ им. Н.Н. Блохина РАМН.-2012.-Т. 23, № 2.- С. 64-80.

8. Нечушкина В.М., Морхов К.Ю., Кузнецов В.В., Егорова А.В. Адъювантная дистанционная лучевая терапия при раке тела матки I - /I стадий // Онкогинекология.-2014.№ 4.- C. 37-44.

9. Aalders J., Abeler V., Kolstad P., Onsrud M. Postoperative external irradiation and prognostic parameters in stage I endometrial carcinoma: clinical and histopathologic study of 540 patients // Obstet. Gynecol.-1980.- Vol. 56, N 4.-P. 419-427.

10. ASTEC study group, Kitchener H., Swart A.M. et al. Efficacy of systematic pelvic lymphadenectomy in endometrial cancer (MRC ASTEC trial): a randomised study // Lancet. - 2009.- Vol. 373, N 9658. - P. 125-136. 
11. Benedetti Panici P., Basile S., Maneschi F. et al. Systematic pelvic lymphadenectomy vs. no lymphadenectomy in early-stage endometrial carcinoma: randomized clinical trial // J. Natl. Cancer Inst.-2008.-Vol. 100, N 23.-P. 1707-1716.

12. Bristow R.E., Zerbe M.J., Rosenshein N.B. et al. Stage IVB endometrial carcinoma: the role of cytoreductive surgery and determinants of survival // Gynecol. Oncol.-2000.-Vol. 78.-P. 85-91.

13. Buda A., Di Martino G., Restaino S. et al. The impact on survival of two different staging strategies in apparent earlystage endometrial cancer comparing sentinel lymph nodes mapping algorithm and selective lymphadenectomy: An Italian retrospective analysis of two reference centers // Gynecol. Oncol._2017._Vol. 147, N 3.P. 528-534.

14. Chan J.K., Cheung M.K., Huh W.K. et al. Therapeutic role of lymph node resection in endometrioid corpus cancer. A Study of 12,333 // Cancer.-2006.-Vol. 107, N 8.-P. 1823-1830.

15. Chan J.K., Urban R., Cheung M.K. et al. Lymphadenectomy in endometrioid uterine cancer staging: how many lymph nodes are enough? A study of 11,443 patients // Cancer.-2007.-Vol. 109, N 12.-P. 2454-2460.

16. Chen J.-R., Chang T.-C., Fu H.-C. et al. Outcomes of patients with surgically and pathologically staged IIIA-IVB pure endometrioidtype endometrial cancer // Medicine.-2016.-Vol. 95.-P. 15.

17. Chino J.P., Jones E., Berchuk A. et al. The influence of radiation modality and lymph node dissection on survival in early-stage endometrial cancer // Int.J. Radiat. Oncol. Biol. Phys.-2012.-Vol. 82, N 5.-P. 1872-1879

18. Colombo N., Creutzberg C., Amant F. et al. ESMO-ESGO-ESTRO consensus conference on endometrial cancer: Diagnosis, treatment and follow-up // Int.J. Gynecol. Cancer.-2016.-Vol. 26, N1.-P. 2-30.

19. Cowles T.A., Magrina J.F., Masterson B.J., Capen C.V. Comparison of clinical and surgical staging in patients with endometrial carcinoma // Obstet. Gynecol.-1985.-Vol. 66, N 3.-P. 413-416.

20. Creasman W.T., Morrow C.P., Bundy B.N. et al. Surgical pathologic spread patterns of endometrial cancer. A Gynecologic Oncology Group Study // Cancer.-1987.-Vol. 60, N8 (suppl.).-P. 2035-2041.

21. Creasman W. T., Odicino F., Maisonneuve P. et al. Carcinoma of the Corpus Uteri // Int.J. Gynaecol. Obstet.-2006.-Vol. 95 (suppl. 1). - P. $5105-S 144$.

22. Creutzberg C.L., van Putten W.L., Koper P.C. et al. Surgery and postoperative radiotherapy versus surgery alone for patients with stage 1 endometrial carcinoma: multicentre randomised trial. PORTEC Study Group. PostOperative Radiation Therapy in Endometrial Carcinoma // Lancet.-2000.-Vol. 355, N 9213.-P. 1404-1411.

23. DiSaia P. J., Creasman W. T. Management of endometrial adenocarcinoma stage I with surgical staging followed by tailored adjuvant radiation therapy // Clin. Obstet. Gynaecol.-1986._Vol. 13, N 4.- P. 751-765.

24. Eto T., Saito T., Kasamatsu T. et al. Clinicopathological prognostic factors and the role of cytoreduction in surgical stage IVB endometrial cancer: A retrospective multi-institutional analysis of 248 patients in Japan // Gynecol. Oncol._2012._Vol. 127.-P. 338-344.
25. Ferlay J., Ervik M., Lam F., et al. Global Cancer Observatory: Cancer Today. [Electronic resource].- Lyon, France: International Agency for Research on Cancer, 2018. - URL: https://gco.iarc.fr/today (дата обращения 24.09.2020).

26. Goff B. A., Riche L.W. Assessment of depth of myometrial invasion in endometrial adenocarcinoma // Gynecol. Oncol.-1990.-Vol. 38, N 1.- P. 46-48.

27. Havrilesky L.J., Cragun J.M., Calingaert B. et al. Resection of lymph node metastases influences survival in stage IIIC endometrial cancer // Gynecol. Oncol.-2005.-Vol. 99, N 3.-P. 689-695.

28. Hirahatake K., Hareyama H., Sakuragi N. et al. A clinical and pathologicstudy on para-aortic lymph node metastasis in endometrial carcinoma // J. Surg. Oncol.-1997.-Vol. 65, N2.P. 82-87.

29. Hsieh C.H., Chang Chien C. C., Lin H. et al. Can a preoperative CA 125 level be a criterion for full pelvic lymphadenectomy in surgical staging of endometrial cancer? // Gynecol. Oncol.-2002.-Vol. 86, N 1.-P. 28-33.

30. Huang C.-Y., Ho C.-M., Chen Y.-L. et al. Impact of lymphadenectomy in uterine endometrioid carcinoma // EJSO.-2013.-Vol. 39.-P. 350-357.

31. Jarvet C.T. The spread of benign and malignant endometrium in the lymphatic system with a note on coexisting vascular involvement // Am.J. Obstet. Gynecol.-1952.-Vol. 64.-P. 780-806.

32. Johnson N., Cornes $P$. Survival and recurrent disease after postoperative radiotherapy for early endometrial cancer: systematic review and meta-analysis // BJOG._2007.-Vol. 114, N11.-P. 1313-1320.

33. Keys H.M., Roberts J.A., Brunetto V.L. et al. A phase III trial of surgery with or without adjunctive external pelvic radiation therapy in intermediate risk endometrial adenocarcinoma: a Gynecologic Oncology Group study // Gynecol. Oncol._2004._Vol. 92, N 3.P. 744-751.

34. Kong A., Johnson N., Kitchener H.C., Lawrie T. A. Adjuvant radiotherapy for stage I endometrial cancer: an updated Cochrane systematic review and meta-analysis / // J. Natl. Cancer Inst.2012.-Vol. 104, N 21. - P. 1625-1634.

35. Lewis B. V., Stallworthy J.A., Cowdell R. Adenocarcinoma of the body of the uterus // J. Obstet. Gynaecol. Br. Commonw.-1970.-Vol. 77, N 4.-P. 343-348.

36. Marziale P., Atlante G., Pozzi M. et al. 426 cases of stage l endometrial carcinoma: a clinicopathological analysis // Gynecol. Oncol.-1989.-Vol. 32, N 3.-P. 278-281.

37. McMeekin D. S., Lashbrook D., Gold M. et al. Analysis of FIGO stage IIIc endometrial cancer patients // Gynecol. Oncol.-2001.-Vol. 81, N 2.-P. 273-278.

38. McMeekin D. S., Lashbrook D., Gold M. Nodal distribution and its significance in FIGO stage III endometrial cancer // Gynecol. Oncol.-2001.-Vol. 82, N 2.-P. 375-379.

39. Nout R.A., Smit V.T. H. B. M., Putter H. et al. Vaginal brachytherapy versus pelvic external beam radiotherapy for patients with endometrial cancer of high-intermediate risk (PORTEC-2): an open-label, non-inferiority, randomised trial // Lancet.-2010.Vol. 375, N 9717.-P. 816-823. 
40. Onda T., Yoshikawa H., Mizutani K. et al. Treatment of node-positive endometrial cancer with complete node dissection, chemotherapy and radiation therapy // Br.J. Cancer.-1997.-Vol. 75, N 12.-P. 1836-1841.

41. Onsrud M., Cvancarova M., Hellebust T.P. et al. Long-term outcomes after pelvic radiation for early-stage endometrial cancer //J. Clin. Oncol.-2013.-Vol. 31, N 31.-P. 3951-3956.

42. Otsuka I., Kubota T., Aso T. Lymphadenectomy and adjuvant therapy in endometrial carcinoma: role of adjuvant chemotherapy // Br.J. Cancer.-2002.-Vol. 87, N 4.-P. 377-380.

43. Shih K.K., Yun E., Gardner G.J. et al. Surgical cytoreduction in stage IV endometrioid endometrial carcinoma // Gynecol. Oncol.2011.-Vol.122.-P. 608-611.
44. StallworthyJ.A. Surgery of endometrial cancer in the Bonney tradition // Ann.R. Coll. Surg. Engl.-1971.-Vol. 48, N 5.-P. 293-305.

45. Straughn J.M., Huh W.K., Kelly F.J. et al. Conservative management of stage l endometrial carcinoma after surgical staging // Gynecol. Oncol.-2002.-Vol. 84, N 2.-P. 194-200.

46. Straughn J. M., Huh W. K., OrrJ.W. et al. Stage IC adenocarcinoma of the endometrium: survival comparisons of surgically staged patients with and without adjuvant radiation therapy // Gynecol. Oncol.-2003.-Vol. 89, N 2.-P. 295-300.

47. Todo Y., Kato H., Kaneuchi M. et al. Survival effect of paraaortic lymphadenectomy in endometrial cancer (SEPAL study): a retrospective cohort analysis // Lancet.-2010.-Vol. 375, N 9721.-P. 1165-1172. 